International Journal of Algebra, Vol. 8, 2014, no. 4, 187 - 194

HIKARI Ltd, www.m-hikari.com

http://dx.doi.org/10.12988/ija.2014.429

\title{
Pure Injective Modules Relative to Torsion Theories
}

\author{
Mehdi Sadik Abbas and Mohanad Farhan Hamid
}

Department of Mathematics, University of Mustansiriya, Baghdad, Iraq

Copyright (c) 2014 Mehdi Sadik Abbas and Mohanad Farhan Hamid. This is an open access article distributed under the Creative Commons Attribution License, which permits unrestricted use, distribution, and reproduction in any medium, provided the original work is properly cited.

\begin{abstract}
Let $\tau$ be a hereditary torsion theory on the category Mod- $R$ of right $R$-modules. A right $R$-module $M$ is called pure $\tau$-injective if it is injective with respect to every pure exact sequence having a $\tau$-torsion cokernel. Every module has a pure $\tau$-injective envelope. A module $M$ is called purely quasi $\tau$-injective if it is fully invariant in its pure $\tau$-injective envelope. A module $M$ is called quasi pure $\tau$-injective if maps from $\tau$ dense pure submodules of $M$ into $M$ are extendable to endomorphisms of $M$. The class of pure $\tau$-injective modules is properly contained in the class of purely quasi $\tau$-injective modules which is in turn properly contained in the class of quasi pure $\tau$-injectives. A torsion theoretic version of each of the concepts of regular and pure semisimple rings is characterized using the above generalizations of pure injectivity.
\end{abstract}

Mathematics Subject Classification: 16D50

Keywords: (quasi) pure injective module, purely quasi injective module, torsion theory

\section{Introduction}

We will denote by $R$ an associative ring with a nonzero identity and by $\tau=$ $(\mathcal{T}, \mathcal{F})$ a hereditary torsion theory on the category Mod- $R$ of right $R$-modules. Unless otherwise stated, all modules considered will be right unital $R$-modules. A submodule $N$ of a module $M$ is said to be $\tau$-dense in $M\left(\operatorname{denoted} N \leq^{\tau d} M\right.$ ) if $M / N$ is $\tau$-torsion. The largest $\tau$-torsion submodule of a module $M$ is denoted $t(M)$ and has the property that $M / t(M)$ is $\tau$-torsion free. The intersection of any finite number of $\tau$-dense submodules is again $\tau$-dense. If $N$ and $K$ are submodules of a module $M$ such that $N \leq^{\tau d} M$ then $N \cap K \leq^{\tau d} K$. 
Any submodule that contains a $\tau$-dense submodule is itself $\tau$-dense. An $R$ module $M$ is called $\tau$-injective if it is injective with respect to every short exact sequence having a $\tau$-torsion cokernel. This is equivalent to saying that every map from a $\tau$-dense right ideal of $R$ to $M$ can be extended to a map $R \rightarrow M$ (generalized Baer criterion). Any module $M$ has a $\tau$-injective envelope, i.e. a $\tau$ injective module that contains $M$ both as $\tau$-dense and an essential submodule. A module $M$ is called $\tau$-quasi injective if every map from a $\tau$-dense submodule of $M$ into $M$ can be extended to an endomorphism of $M$. This is equivalent to saying that $M$ is fully invariant in its $\tau$-injective envelope. For preliminaries about torsion theories, we refer to [3].

A short exact sequence $0 \rightarrow N^{\prime} \rightarrow N \rightarrow N^{\prime \prime} \rightarrow 0$ of right $R$-modules is called pure exact if it remains exact when tensored with any (finitely presented) left $R$-module [5]. A module $M$ is called $N$-pure injective if it is injective with respect to every pure exact sequence $0 \rightarrow N^{\prime} \rightarrow N \rightarrow N^{\prime \prime} \rightarrow 0$. The module $M$ is called pure injective if it is $N$-pure injective for every $R$-module $N$ if and only if it is a direct summand of every module containing it as a pure submodule. The module $M$ is called quasi pure injective if it is $M$ pure injective [8]. Given a class of modules $\mathcal{X}$, a map $f: M \rightarrow X$, where $X \in \mathcal{X}$, is called an $\mathcal{X}$-preenvelope of the module $M$ if for any $X^{\prime} \in \mathcal{X}$ every map $f^{\prime}: M \rightarrow X^{\prime}$ factors through $X$. If furthermore, when $X=X^{\prime}$ and $f=f^{\prime}$, the only such factorizations are automorphisms we say that $f$ : $M \rightarrow X$ is an $\mathcal{X}$-envelope. If $f: M \rightarrow X$ is an $\mathcal{X}$-envelope we sometimes say that $X$ is an $\mathcal{X}$-envelope of $M$. If every module has an $\mathcal{X}$-(pre)envelope we say that the class $\mathcal{X}$ is (pre)enveloping. An envelope of a module when it exists is unique up to isomorphism (p.129 of [6]). The class of pure injective modules is enveloping [9]. The pure injective envelope of a module $M$ is denoted $P E(M)$. A module is called purely quasi injective if it is fully invariant in its pure injective envelope [2]. Every purely quasi injective module is quasi pure injective but not conversely [2].

In this paper we generalize the above concepts to a torsion theoretic setting. We say that a module is pure $\tau$-injective if it is injective with respect to every pure exact sequence that has a $\tau$-torsion cokernel. We will prove that the class of pure $\tau$-injective modules is enveloping. Then we study modules that are fully invariant in their pure $\tau$-injective envelopes. The last section is about caharacterizing certain types of rings in terms of pure $\tau$-injective and purely quasi $\tau$-injective modules.

\section{Relative Pure $\tau$-Injectivity}

Definition 2.1 Let $M$ and $N$ be right $R$-modules. The module $M$ is called:

1. $N$-pure $\tau$-injective if $M$ is injective with respect to all pure exact se- 


$$
\text { quences } 0 \rightarrow N^{\prime} \rightarrow N \rightarrow N / N^{\prime} \rightarrow 0 \text { such that } N / N^{\prime} \text { is } \tau \text {-torsion. }
$$

2. quasi pure $\tau$-injective if $M$ is $M$-pure $\tau$-injective. pure $\tau$-injective if it is $N$-pure $\tau$-injective for every $R$-module $N$.

Of course the class of $N$-pure $\tau$-injective modules is closed under direct products, direct summands and finite direct sums. Any $N$-pure injective module is $N$-pure $\tau$-injective. If $\tau$ is the torsion theory in which every $R$-module is $\tau$-torsion then a module is $N$-pure $\tau$-injective if and only if it is $N$-pure injective.

For the next Theorem, consider the following condition on a module $N$ and an exact sequence $0 \rightarrow M \stackrel{\alpha}{\longrightarrow} P$ :

$\left(^{*}\right)$ There is a map $\beta: N \rightarrow P$ such that $P=\alpha(M)+\beta(N)$ and $\beta^{-1}(\alpha(M))$ is a pure and $\tau$-dense submodule of $N$.

Theorem 2.2 For $R$-modules $M$ and $N$, the following are equivalent:

1. $M$ is $N$-pure $\tau$-injective.

2. Every exact sequence $0 \rightarrow M \stackrel{\alpha}{\longrightarrow} P$ that satisfies condition (*) splits.

3. Every pure exact sequence $0 \rightarrow M \stackrel{\alpha}{\longrightarrow} P$ that satisfies condition (*) splits.

4. Every exact sequence $0 \rightarrow M \stackrel{\alpha}{\longrightarrow} P$ that satisfies condition (*) and has a $\tau$-torsion cokernel splits.

5. Every pure exact sequence $0 \rightarrow M \stackrel{\alpha}{\longrightarrow} P$ that satisfies condition (*) and has a $\tau$-torsion cokernel splits.

Proof. (1) $\Rightarrow(2)$ Put $K=\beta^{-1}(\alpha(M))$. By hypothesis the map $\alpha^{-1} \beta: K \rightarrow M$ extends to $\gamma: N \rightarrow M$. By assumption $P=\alpha(M)+\beta(N)$, i.e. for each $x \in P$ there exist $m$ and $n$ in $M$ and $N$ respectively, such that $x=\alpha(m)+\beta(n)$. Define $\delta: P \rightarrow M$ by $\delta(x)=m+\gamma(n)$. To prove $\delta$ is well-defined let $x=$ $\alpha(m)+\beta(n)=\alpha\left(m^{\prime}\right)+\beta\left(n^{\prime}\right)$, hence $\alpha\left(m-m^{\prime}\right)=\beta\left(n^{\prime}-n\right) \in \alpha(M) \cap \beta(N)$ so that $m-m^{\prime}=\alpha^{-1} \beta\left(n^{\prime}-n\right)=\gamma\left(n^{\prime}-n\right)$. Thus $m+\gamma(n)=m^{\prime}+\gamma\left(n^{\prime}\right)$. It is clear that $\delta \alpha=1_{M} .(2) \Rightarrow(3) \Rightarrow(5)$ and $(2) \Rightarrow(4) \Rightarrow(5)$ are trivial. $(5) \Rightarrow(1)$ Let $K$ be a pure $\tau$-dense submodule of $N$ and $f: K \rightarrow M$ be a given map and form the pushout diagram:

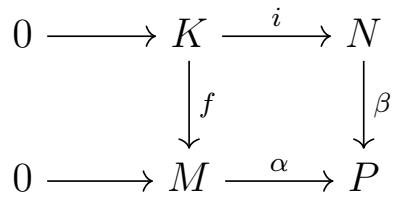


where $P=(M \oplus N) / W$, with $W=(k, f(k)), k \in K$. If the second row splits then we get a map $\alpha^{\prime}: P \rightarrow M$ such that $\alpha^{\prime} \alpha=1_{M}$, hence $\alpha^{\prime} \alpha i=f$ gives an extension of $f$. So we need to show that condition $\left(^{*}\right)$ together with the conditions in (5) hold. Now as the diagram is a pushout and the upper sequence is pure exact with a $\tau$-torsion cokernel, so is the lower sequence. But it is easy to see that $P=\alpha(M)+\beta(N)$ and $\beta^{-1}(\alpha(M))=K$ which is pure and $\tau$-dense in $N$. Hence the result.

Corollary 2.3 A module is pure $\tau$-injective if and only if it is a direct summand of every module containing it as a pure $\tau$-dense submodule.

The following Proposition shows that the class of pure $\tau$-injective modules is preenveloping.

Proposition 2.4 Every module can be embedded as a pure $\tau$-dense submodule of a pure $\tau$-injective module. Such an embedding is a pure $\tau$-injective preenvelope.

Proof. Consider $t(P E(M) / M)$, the $\tau$-torsion part of $P E(M) / M$ and let $P$ be the complete inverse image of $t(P E(M) / M)$ under the natural map $P E(M) \rightarrow$ $P E(M) / M$. It is clear that $P$ is a submodule of $P E(M)$ containing $M$ as a pure $\tau$-dense submodule and that $P E(M) / P$ is $\tau$-torsion free. Now consider the row exact commutative diagram:

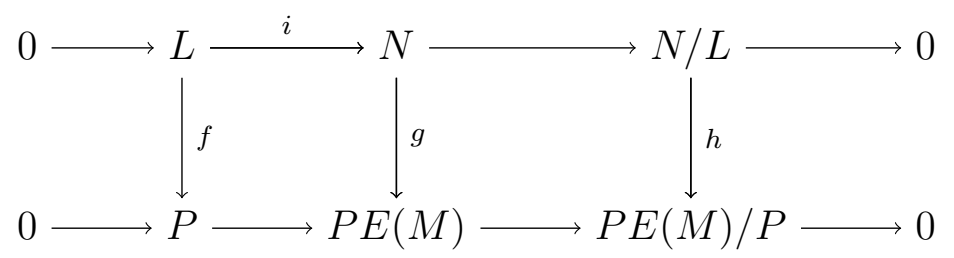

where $L$ is $\tau$-dense and pure in $N, f$ is a given map, $g$ is the map whose existence is ensured by pure injectivity of $P E(M)$ and $h$ is the map defined by $x+L \mapsto g(x)+P$. Since $N / L \in \mathcal{T}$ and $P E(M) / P \in \mathcal{F}$ we must have $h=0$. Hence $g(N) \subseteq P$ and so $P$ is pure $\tau$-injective. It remains to show that $P$ is a pure $\tau$-injective preenvelope of $M$. But any map $M \rightarrow P^{\prime}$ into a pure $\tau$-injective module $P^{\prime}$ can be extended to a map $P \rightarrow P^{\prime}$ since $M$ is pure and $\tau$-dense in $P^{\prime}$.

Next we show that every module admits a pure $\tau$-injective envelope, but before this we need a lemma due to Enochs and Jenda (p.139 of [6]):

Lemma 2.5 Let $\mathcal{X}$ be a class of $R$-modules that is closed under summands, and $M$ an $R$-module. Suppose that $M$ has an $\mathcal{X}$-preenvelope and if $\left(\left(X_{i}\right)\right.$, $\left.\left(\phi_{j i}\right)\right)$ is any well-ordered inductive system of $\mathcal{X}$-preenvelopes of $M$, then for some $\mathcal{X}$-preenvelope $M \rightarrow X$ there is a factorization $M \rightarrow \underset{\longrightarrow}{\longrightarrow} X_{i} \rightarrow X$. Then $M$ has an $\mathcal{X}$-envelope. 
Theorem 2.6 Every module has a pure $\tau$-injective envelope.

Proof. Given an inductive system $\left(P_{i},\left(\phi_{j i}\right)\right)$ of pure $\tau$-injective envelopes of a module $M$, the following sequences

$$
0 \rightarrow M \rightarrow P_{i} \rightarrow P_{i} / M \rightarrow 0
$$

are all pure exact with $\tau$-torsion cokernels. The limit of this system of sequences is again pure exact with a $\tau$-torsion cokernel

$$
0 \rightarrow M \rightarrow \underset{\longrightarrow}{\lim } P_{i} \rightarrow \lim _{\longrightarrow} P_{i} / M \rightarrow 0
$$

Hence any map $M \rightarrow P$ into a pure $\tau$-injective preenvelope of $M$ is extendable to a map $\lim P_{i} \rightarrow P$. So $M$ has a pure $\tau$-injective envelope by Lemma 2.5 .

Let us denote the pure $\tau$-injective envelope of the module $M$ by $P_{\tau}(M)$. We can now study modules that are fully invariant in their pure $\tau$-injective envelopes, compare with [2].

Definition 2.7 Let $M$ and $N$ be right $R$-modules. The module $M$ is called:

1. Purely $N$ - $\tau$-injective if for any map $f: P_{\tau}(N) \rightarrow P_{\tau}(M)$, we have $f(N) \subseteq M$.

2. Purely quasi $\tau$-injective if $M$ is purely $M$ - $\tau$-injective, i.e. if $M$ is fully invariant in its pure $\tau$-injective envelope.

It is easy to see that a module $M$ is purely $N$ - $\tau$-injective if and only if for any map $f: N \rightarrow P_{\tau}(M)$, we have $f(N) \subseteq M$. Any purely $N$-injective is purely $N$ - $\tau$-injective. If $\tau$ is the improper torsion theory then a module $M$ is purely $N$ - $\tau$-injective (respectively, purely quasi $\tau$-injective) if and only if $M$ is purely $N$-injective (respectively, purely quasi injective).

Every pure $\tau$-injective (and hence every pure injective) module is purely $N$ - $\tau$-injective for every $R$-module $N$. The converse is not true in general as one may take a von Neumann regular ring $R$ that is not noetherian so that there is a quasi injective $R$-module which is not injective [4]. Certainly this serves as a counter example where $\tau$ is the improper torsion theory. Another example for a ring which is not regular is example 2 of [2].

Proposition 2.8 For any two modules $M$ and $N$, if $M$ is purely $N$ - $\tau$-injective then $M$ is $N$-pure $\tau$-injective.

Proof. Let $f: K \rightarrow M$ be a homomorphism from a pure $\tau$-dense submodule of $N$ into $M$. Therefore $f$ can be extended to a map $g: N \rightarrow P_{\tau}(M)$ and hence by assumption, $g(N) \subseteq M$. Hence $g$ is the desired extension of $f$.

Corollary 2.9 Any purely quasi $\tau$-injective module is quasi pure $\tau$-injective. 
The converse of corollary 2.9 (and Proposition 2.8) is not true in general as in the following example which is given with a different proof in example 3 of $[2]$ :

Example 2.10 It is easy to check that $\mathbb{Z}$ is quasi pure injective but not pure injective hence quasi pure $\tau$-injective but not pure $\tau$-injective, where $\tau$ is the improper torsion theory on Mod- $\mathbb{Z}$. But $\mathbb{Z}$ is not fully invariant in $P E(\mathbb{Z})$. Indeed if we take any element $a$ in $P E(\mathbb{Z})$ that is not in $\mathbb{Z}$ and define a map $\mathbb{Z} \rightarrow P E(\mathbb{Z})$ by $1 \mapsto a$ which extends to an endomorphism of $P E(\mathbb{Z})$ then the image of $\mathbb{Z}$ under this extension, is not contained in $\mathbb{Z}$.

Proposition 2.11 The direct sum $M_{1} \oplus M_{2}$ of two modules $M_{1}$ and $M_{2}$ is purely quasi $\tau$-injective if and only if $M_{i}$ is purely $M_{j}-\tau$-injective for each $i, j=1,2$.

Proof. Suppose that $M_{1} \oplus M_{2}$ is purely quasi $\tau$-injective. For arbitrary $i$ and $j$, let $f: M_{i} \rightarrow P_{\tau}\left(M_{j}\right)$ be a map. Now $f$ can be easily extended to a map $M_{1} \oplus M_{2} \rightarrow P_{\tau}\left(M_{1}\right) \oplus P_{\tau}\left(M_{2}\right)$ which we again call it $f$. By assumption $f\left(M_{1} \oplus M_{2}\right) \subseteq M_{1} \oplus M_{2}$. Hence $f\left(M_{i}\right) \subseteq M_{j}$. Conversely let $f: M_{1} \oplus M_{2} \rightarrow$ $P_{\tau}\left(M_{1} \oplus M_{2}\right)$ be a map. Restrict $f$ to $M_{i}$ and follow it by the projection $M_{1} \oplus M_{2} \rightarrow M_{j}$ to get a map $f_{i j}: M_{i} \rightarrow M_{j}$. Since $f_{i j}\left(M_{i}\right) \subseteq M_{j}$, we must have $f\left(M_{1} \oplus M_{2}\right) \subseteq M_{1} \oplus M_{2}$.

\section{Characterization of Rings}

Regular rings $R$ are characterized by any one of the following:

1. Every ideal of $R$ is pure. [7]

2. Every exact sequence of $R$-modules is pure exact. [7]

3. Every pure injective $R$-module is injective. [10]

4. Every purely quasi injective $R$-module is quasi injective. [2]

5. Every quasi pure injective $R$-module is quasi injective. [1]

Here we give a torsion theoretic version of the above characterizations.

Lemma 3.1 For an exact sequence $0 \rightarrow K \rightarrow L \rightarrow N \rightarrow 0$ such that $N$ is $\tau$-torsion, the following statements are equivalent:

1. The sequence is pure exact.

2. Every pure $\tau$-injective $R$-module is injective with respect to the given sequence. 
Proof. $(1) \Rightarrow(2)$ Clear. (2) $\Rightarrow$ (1) In particular, every pure injective $R$-module is injective with respect to the sequence, so by 34.7 of [10] the result follows.

Theorem 3.2 For a ring $R$ the following statements are equivalent:

1. Any exact sequence $0 \rightarrow I \rightarrow R \rightarrow R / I \rightarrow 0$ such that $R / I$ is $\tau$-torsion, is pure exact.

2. Any exact sequence $0 \rightarrow K \rightarrow L \rightarrow N \rightarrow 0$ such that $N$ is $\tau$-torsion, is pure exact.

3. Every pure $\tau$-injective $R$-module is $\tau$-injective.

4. Every pure $\tau$-injective $R$-module is $\tau$-quasi injective.

5. Every purely quasi $\tau$-injective $R$-module is $\tau$-quasi injective.

6. Every quasi pure $\tau$-injective $R$-module is $\tau$-quasi injective.

Proof. $\quad(2) \Rightarrow(1) \Rightarrow(3) \Rightarrow(4)$ and $(2) \Rightarrow(6) \Rightarrow(5) \Rightarrow(4)$ are trivial. $(3) \Rightarrow(2)$ is clear by Lemma 3.1. (4) $\Rightarrow(3)$ Let $M$ be a pure $\tau$-injective $R$-module and put $P=P_{\tau}(R)$ so that $M \oplus P$ is pure $\tau$-injective, and hence $\tau$-quasi injective by assumption. If we could show that $M$ is $\tau$-injective with respect to $R$ then it would be $\tau$-injective by the generalized Baer criterion. So let $I \leq \leq^{\tau d} R$ hence $I \leq^{\tau d} P$ and then $M \oplus I \leq^{\tau d} M \oplus P$. Now any map $f: I \rightarrow M$ can be easily extended to a map $M \oplus I \rightarrow M \oplus P$ given by $(m, i) \mapsto(f(i), 0)$. Since $M \oplus P$ is $\tau$-quasi injective this map is extendable to an endomorphism of $M \oplus P$. Restrict this endomorphism to $R$ and follow it by the projection map $M \oplus P \rightarrow M$ to get the desired extension of $f$.

Next, we give torsion theoretic version of pure semisimple rings. Recall that a ring $R$ is called pure semisimple if every pure exact sequence of $R$ modules splits. Pure semisimple rings are characterized by the property that every module is pure injective (see p.529 of [10]). This is equivalent to saying that every $R$-module is purely quasi injective [2], which is equivalent to the statement that every $R$-module is quasi pure injective [1]. Now by a $\tau$-pure semisimple ring we mean a ring $R$ over which every pure exact sequence of $R$-modules with $\tau$-torsion cokernel splits.

Theorem 3.3 For a ring $R$ the following are equivalent:

1. $R$ is $\tau$-pure semisimple.

2. Every $R$-module is pure $\tau$-injective.

3. Every R-module is purely quasi $\tau$-injective. 
4. Every $R$-module is quasi pure $\tau$-injective.

Proof. $(2) \Rightarrow(1)$ and $(2) \Rightarrow(3) \Rightarrow(4)$ are obvious. (1) $\Rightarrow(2)$ Let $M$ be an $R$-module. Now $0 \rightarrow M \rightarrow P_{\tau}(M) \rightarrow P_{\tau}(M) / M \rightarrow 0$ is a pure exact sequence with $P_{\tau}(M) / M \in \mathcal{T}$, hence the sequence splits, which means that $M$ is pure $\tau$-injective. $(4) \Rightarrow(2)$ For any $R$-module $M$ with $P=P_{\tau}(M)$, we have $M \oplus P$ is quasi pure $\tau$-injective by assumption. Consider the map $M \oplus M \rightarrow M \oplus P$ given by $\left(m^{\prime}, m\right) \mapsto(m, 0)$. Since $M \oplus M$ is both pure and $\tau$-dense in $M \oplus P$, the above map extends to an endomorphism of $M \oplus P$. Restrict this endomorphism on $P$ and follow it by the projection $M \oplus P \rightarrow M$ to get a map $P \rightarrow M$ that extends the identity map of $M$. Hence $M$ is a direct summand of $P$.

Acknowledgements. Thanks are due to Professor E. Enochs for reading the manuscript and providing his helpful remarks.

\section{References}

[1] M. S. Abbas, M. F. Hamid, On Relative Pure Injective Modules. AlMustansiriyah J. Sci., 21 (6) (2010), 374-382.

[2] M. S. Abbas, M. F. Hamid, (Flat) Modules that are Fully Invariant in Their Pure-Injective (Cotorsion) Envelopes. Al-Mustansiriyah J. Sci., 22 (4) (2011), 351-359.

[3] P. E. Bland, Topics in Torsion Theory. Wily-VCH, Berlin (1998).

[4] K. A. Byrd, Rings whose quasi-injective modules are injective, Proc. Amer. Math. Soc. 33 (2) (1972), 235-240.

[5] P. M. Cohn, On the free product of associative rings. I. Math. Z. 71 (1959), 380-398.

[6] E. E. Enochs, O. M. G. Jenda, Relative Homological Algebra. De Gruyter Expositions in Mathematics 30, Berlin (2000).

[7] D. Fieldhouse, Pure theories. Math. Ann. 184 (1969), 1-18.

[8] L. X. Mao, N. Q. Ding, Cotorsion modules and relative pure injectivity, J. Aust. Math. Soc. 81 (2006) 225-243.

[9] R. B. Warfield, Purity and algebraic compactness for modules. Pacific J. Math. 28 (3) (1969), 699-719.

[10] R. Wisbauer, Foundations of Module and Ring Theory. Gordon and Breach, 1991.

Received: February 6, 2014 